\title{
Non-invasive brain stimulation for the treatment of brain diseases in childhood and adolescence: state of the art, current limits and future challenges
}

\author{
Carmelo M. Vicario ${ }^{1 *}$ and Michael A. Nitsche ${ }^{2}$ \\ 1 School of Psychology, The University of Queensland, St. Lucia, QLD, Australia \\ ${ }^{2}$ Clinic for Clinical Neurophysiology, University Medical Center, Göttingen, Germany
}

Edited by:

Mikhail Lebedev, Duke University, USA

\section{Reviewed by:}

Yoshio Sakurai, Kyoto University, Japan

Estate M. Sokhadze, University of Louisville, USA

Kyongsik Yun, California Institute of Technology, USA

\section{*Correspondence:}

Carmelo M. Vicario, School of

Psychology, The University of

Queensland, McElwain Building, St.

Lucia, QLD 4072, Australia

e-mail: carmelo.vicario@uniroma1.it
In the last decades interest in application of non-invasive brain stimulation for enhancing neural functions is growing continuously. However, the use of such techniques in pediatric populations remains rather limited and mainly confined to the treatment of severe neurological and psychiatric diseases. In this article we provide a complete review of non-invasive brain stimulation studies conducted in pediatric populations. We also provide a brief discussion about the current limitations and future directions in a field of research still very young and full of issues to be explored.

Keywords: pediatric brain stimulation, vascular diseases, epilepsy, ADHD, Tourette, autism, depression, schizophrenia

\section{INTRODUCTION}

In the last decades the interest in exploring therapeutic and/or rehabilitative effects generated by non-invasive brain stimulation techniques in neuropsychiatric diseases increased considerably. Although this field of research encompasses numerous stimulation techniques, neuroscientists have primarily focused investigations on the use of two techniques, namely Transcranial Magnetic Stimulation (TMS) and transcranial Direct Current Stimulation (tDCS). In healthy adults, these non-invasive brain stimulation techniques are applied to monitor cortical excitability/dynamics (e.g., single pulse TMS, double pulse TMS), and as neuromodulatory techniques [e.g., repetitive TMS (rTMS), tDCS]. Whereas TMS is primarily used to investigate brain physiology (e.g., task-dependent alterations of cortical maps, excitability, and so on, in health, and disease), the latter techniques are applied to modify physiology, and performance. Accordingly, these methods have been adopted to explore, and modulate brain functions such as language (e.g., rTMS: Flöel et al., 2008; tDCS: Vicario and Rumiati, 2012;), learning, and long-term memory formation [e.g., single pulse TMS (spTMS): Vicario et al., 2013a; tDCS: Nitsche et al., 2003], working memory (WM) (e.g., TMS: Gaudeau-Bosma et al., 2013; tDCS: Fregni et al., 2005a) perception (e.g., TMS: Hamilton et al., 2013; tDCS: Vicario et al., 2013b), and attention (e.g., TMS: Lee et al., 2013; tDCS: Tanoue et al., 2013), in healthy humans (see Nitsche et al., 2003, 2008; Kuo and Nitsche, 2012 for further examples and Nitsche and Paulus, 2011 for a complete review).

Non-invasive brain stimulation is applied also to rehabilitate cortical functions in neuropsychiatric diseases via induction of neuroplasticity. rTMS has been shown to improve cognitive functions in Parkinson's disease (Pascual-Leone et al., 1994), and improved aphasia (Naeser et al., 2005), motor control after stroke (Takeuchi et al., 2005), epilepsy (see Nitsche and Paulus, 2009 for a review), and depression (Pascual-Leone et al., 1996; O'Reardon et al., 2007), amongst others (Kammer and Spitzer, 2012).

Encouraging clinical effects have been documented also for tDCS, so far in pilot studies. tDCS improves motor and nonmotor stroke symptoms (e.g., Fregni et al., 2005b), depression, and might have effects on craving, schizophrenia, and dementia, amongst others (for comprehensive reviews, see, Flöel, 2013; Krause et al., 2013; Kuo et al., 2013). All these results encourage the future therapeutic application of noninvasive brain stimulation techniques also in pediatric populations affected by brain disorders. However, the number of non-invasive brain stimulation studies conducted in childhood is scanty, especially if compared to that available from adult participants.

Two previous reviews (Quintana, 2005; Croarkin et al., 2011) gave an overview of the application of rTMS in children and adolescents. Here, we provide the reader with an updated state of the art of application of non-invasive brain stimulation in general (rTMS and tDCS) in pediatric populations. Moreover, we will discuss current limits and possible future applications of these techniques for the treatment of brain dysfunctions affecting childhood. It is beyond the scope of this review to discuss the mechanisms of how TMS and tDCS alter neural activity and induce brain plasticity in detail. For obtaining this information, some recently published articles provide a complete and exhaustive overview (see for example Stagg and Nitsche, 2011; Freitas et al., 2013; Krause et al., 2013). 


\section{NON-INVASIVE BRAIN STIMULATION FOR ENHANCING BRAIN DISORDERS IN CHILDHOOD VASCULAR DISEASES}

Pathophysiologically, stroke is associated with hemispheric dysbalance, i.e., a reduction of the activity of the lesioned brain area and an enhanced activity of the contra-lesional homologous region, which limits re-gain of functions (see Grefkes and Fink, 2011 for a recent review).

Kirton et al. (2008) conducted the first randomized shamcontrolled rTMS trial in children (median age 13.25) affected by arterial ischemic stroke. Patients were affected by unilateral hand motor weakness. They received 8 days to $1 \mathrm{~Hz}$ rTMS of the contralesional motor cortex, which inhibits regional brain activity (Pascual-Leone et al., 2005) and increases contralateral cortical excitability via reduction of interhemispheric inhibition (Pal et al., 2005). Grip strength increased after real rTMS only, and this effect persisted until day 17 after the start of treatment. Furthermore, rTMS was well tolerated with no relevant side effects.

Valle and colleagues (2007) applied a 5-day course of rTMS upon the affected motor cortex in seventeen children (mean age 9) affected by infantile cerebral palsy and spastic quadriplegia. The study was designed according to the hypothesis that an increase of motor cortex activity would increase its inhibitory influence on spinal excitability, and thus improve spasticity. Patients received five consecutive sessions of rTMS in a randomized, sham controlled, double-blind, parallel, clinical trial design. According to previous works (e.g., Quartarone et al., 2005) showing that $5 \mathrm{~Hz}$ rTMS of the primary motor cortex induces an overall increase of excitability of the corticospinal output system, including spinal motoneurons, the authors report a significant reduction of spasticity only in association to this stimulation protocol. Both studies show a potential of rTMS for rehabilitation of motor symptoms originating from vascular injuries in childhood. However, due to the limited number of studies, we are still far from achieving a clear picture of the use of this technique to treat vascular problems in children. No studies for the treatment of vascular disorders in children or adolescents have been conducted with tDCS so far.

\section{EPILEPSY}

The pathophysiological substrate of epilepsies and the proneness to develop seizures is an enhanced cortical excitability, leading to paroxysmal depolarization shifts, an enhanced probability of high-frequent and hyper-synchronous activity of neuronal networks (Stafstrom, 2006; Dudek and Sutula, 2007). A reduction of neuronal excitability is the common aim of antiepileptic therapies.

Fregni et al. (2005c), tested the effect of a single $0.5 \mathrm{~Hz}$ rTMS session in an open study. Three pediatric patients with focal epilepsy were included. The TMS coil was positioned over the epileptogenic region, or, if this could not be clearly identified, over Cz. rTMS significantly reduced the frequency of epileptiform discharges (ED) for up to 15 and 30 days after rTMS treatment.

The study of Brasil-Neto et al. (2004) included a 6 years old patient. This patient was affected by left fronto-central slow activity. $0.3 \mathrm{~Hz}$ rTMS was applied once per day for 3 months. In this case, rTMS treatment was ineffective. Graff-Guerrero et al. (2004) conducted one session of $20 \mathrm{~Hz}$ rTMS in two patients (7 and 11 years old) suffering from epilepsia partialis continua (EPC). The patients were first submitted to a single photon emission computed tomography (SPECT) session in order to localize the focal frontal hyperperfused region to define the stimulation site; after rTMS, patients were SPECT-monitored again in order to identify perfusion alterations of the stimulated region. Indeed, cortical perfusion of the stimulated area was reduced in both patients. However, only in one patient epileptic seizures decreased significantly. Morales et al. (2005) conducted a case study involving 2 patients ( 8 and 16 years old) affected by EPC. One Hertz/six Hertz rTMS were applied in different sessions. No adverse effects occurred, but the treatment resulted in no clinical effects. Kinoshita et al. (2005) treated a 16 years old male suffering from parietal lobe epilepsy with $0.9 \mathrm{~Hz}$ rTMS over 5 days. The coil was placed over PCz. No significant improvements have been documented for this patient.

San-Juan et al. (2011) applied cathodal tDCS over the F2 scalp site in a 17 years old patient affected by Rasmussen's encephalitis [ $4 \mathrm{tDCS}$ sessions of $60 \mathrm{~min}$ (on days $0,7,30$, and 60)]. At follow-up evaluations 6 and 12 months later, seizure frequency was significantly reduced. Additionally, the patient had improved levels of alertness and language. No side effects have been reported. Yook et al. (2011) applied cathodal tDCS applied upon the epileptogenic focus in a 11 year's old patient. The patient was diagnosed with congenital bilateral perisylvian syndrome. Cathodal tDCS was applied over the right temporoparietal area that showed epileptiform activity in the EEG for 2 weeks. During the two-month period after treatment termination, only six seizures occurred (compared to eight seizures a month before the treatment), and seizure duration decreased. tDCS was repeated for another 2 weeks, 2 months after the first intervention session. For the following two months, only one seizure occurred. No notable side effects of stimulation were observed.

In contrast, in a group of pediatric patients (age range 6-11) showing continuous spikes and slow waves during sleep (CSWS), one session of cathodal tDCS over the peak negativity of the epileptogenic pattern revealed an effect on EEG patterns only in 3 patients (Varga et al., 2011). One reason for this negative result might be that the multifocal/diffuse and poorly defined origin of epileptic activity in CSWS makes it difficult to identify the optimal region for stimulation (Brazzo et al., 2012). Moreover, stimulation was performed with $1 \mathrm{~mA}$ in only one session in the latter study, which might have not been sufficient to obtain significant or lasting effects.

More recently, Auvichayapat et al. (2013) enrolled thirty six children (age range 6-15 years) with focal epilepsy in a crossover sham-controlled study. Participants received a single session of cathodal tDCS upon the epileptogenic region. Active tDCS treatment was associated with significant reductions in epileptic discharge frequency immediately and 24 and $48 \mathrm{~h}$ after tDCS. Moreover, 4 weeks after treatment, a small (clinically negligible but statistically significant) decrease in seizure frequency was detected. All patients tolerated tDCS well. 
These studies deliver some preliminary hints for a possible efficacy of rTMS, and tDCS, to treat epilepsy in children. However, the heterogeneity of the results, possibly due to different stimulation parameters, small numbers, and different etiologies of participants, and the heterogeneous quality of the studies, do not allow to draw definite conclusions.

\section{ADHD}

Attention-deficit/hyperactivity disorder (ADHD) is a highly prevalent and impairing disorder, characterized by inattention, hyperactivity, and executive dysfunction. Functional neuroimaging studies have shown functional and abnormalities in cingulate, frontal and parietal cortical regions, including hypoactivation (Bush, 2011). Thus, non-invasive brain stimulation procedures to improve ADHD symptoms are oriented toward excitatory protocols.

Weaver et al. (2008) performed a randomized, shamcontrolled, crossover study involving 9 participants affected by ADHD. The age range of the 4 included adolescents was between 15 and 17 years. $10 \mathrm{~Hz}$ rTMS over 2 weeks was applied upon the right DLPFC. Clinical global impression and the ADHD-IV scales improved significantly, but similarly for active and sham rTMS. No serious adverse effects did take place. No studies on childhood ADHD have been performed with tDCS so far.

\section{TOURETTE SYNDROME}

Tourette's syndrome (TS) is one of the most common neurobehavioral disorders in childhood (see American Academy of Pediatrics, 2000). The pathology is characterized by the presence of tics, which are rapid, stereotyped movements and vocalizations, virtually involving all body segments (Vicario et al., 2010). The neuro-functional profile of childhood TS is characterized by impairment of neural circuits linking the cerebral cortex to the striatum and other sub-cortical regions (Swain et al., 2007; Bush, 2011).

Of interest for the current discussion is the Supplemental Motor Area (SMA). The pre-motor cortex has been reported to be hyperexcitable in patients with TS (George et al., 2001). Therefore, excitability-reducing rTMS to the SMA may be an effective way to treat TS, because this region is extensively connected with regions implicated in motor control (Picard and Strick, 2001).

In an open label 12 weeks cohort pilot study (Kwon et al., 2011), 1Hz rTMS was applied over the SMA for 10 days. At the end of each day subjects completed objective ratings of ADHD, mood, anxiety, tics, and side effects. Statistically significant reductions were seen in the Yale Global TS Severity Scale over the 12 weeks of the study. Le et al. (2013) tested the effect of $1 \mathrm{~Hz}$ rTMS upon the SMA in 25 children with TS (aged less than 16 years) for 20 days. Results document a significant improvement of clinical symptoms. Interestingly, the benefits lasted for up to 6 months in $68 \%$ of subjects. No studies for TS treatment have been conducted with tDCS. The results provided in these two studies are coherent in terms of therapeutic benefits, stimulation site (SMA) and rTMS frequency $(1 \mathrm{~Hz})$. However, an important limit of these studies is the lack of a control group.

\section{AUTISM SPECTRUM DISORDER (ASD)}

This syndrome is characterized by a marked decrease in social integration and communication, and affects $\sim 1$ in 150 children (Fombonne, 2009). Increased gamma-band responses to several cognitive processes in children with autism spectrum disorder have been described (McFadden et al., 2012). While its precise role is unclear, it is implied in a wide range of processes such as attention (Lakatos et al., 2008), WM (Tallon-Baudry et al., 1998), and language (Tavabi et al., 2011). Non-invasive brain stimulation has been adopted for modulating this physiological parameter and therefore improve the related cognitive abilities.

Baruth et al. (2010) report clinical improvements in pediatric ASD induced via rTMS $(\leq 1 \mathrm{~Hz})$ in a controlled study, the electrophysiological effects of 12 low frequency rTMS sessions, bilaterally applied to the DLPFC were explored in 25 subjects (ages range 9-26) with ASD and 20 age-matched controls. rTMS was administered once per week. The first six treatments were performed over the left, and the remaining six over the right DLPFC. Patients showed significant improvement in discriminatory gamma activity and also significant improvements in behavioral parameters. In another study (Sokhadze et al., 2010) 20 subjects (age range 10-19) with ASD received the same protocol. Performance was tested with the oddball paradigm, which explores attentional shifting (García-Larrea et al., 1992). rTMS minimized early cortical responses to irrelevant stimuli, increased responses to relevant stimuli, reduced the error percentage and repetitive-ritualistic behavior.

Schneider and Hopp (2011) conducted an open tDCS study in children with autism. The purpose was to improve language acquisition in patients with minimal verbal language. In this study the authors selected 10 ASD participants (age range 16-21). Postanodal tDCS of the Broca area, mean vocabulary scores were significantly higher than the pre-anodal tDCS scores.

The studies are suggestive for therapeutic benefits of noninvasive brain stimulation in autistic children. However, due to the small numbers of studies, in which different protocols had effects on different target functions, statements about optimal protocols are premature.

\section{DEPRESSION}

Depression in children and adolescents is associated with significant functional disability in multiple environmental realms (Cosgrove et al., 2013). Depression involves a distributed network of cortical and limbic regions, including the DLPFC (especially the left), and subgenual cingulate gyrus, amongst others (Mayberg, 2007). It has been shown (Fitzgerald et al., 2006) that in depression these areas, and specifically the left-hemispherical ones, may be hypoactive, whereas right-hemispheric hyperactivation might take place, thus constituting an hemispheric dysbalance of activation. The rationale of the brain stimulation studies presented here is to increase the activity of the left DLPFC.

Walter et al. (2001) were the first to report the impact of rTMS on depression in 3 patients younger than 18 years. The patients received daily treatment over 2 weeks of $10 \mathrm{~Hz}$ rTMS over the left DLPFC. Two participants improved clinically, but one of them complained about tension headache during two of the treatment sessions. In another case report series, Loo et al. 
(2006) tested the effect of $10 \mathrm{~Hz}$ rTMS upon the left DLPFC over 6 weeks on two 16-year-old adolescent girls affected by depression and ADHD. No improvements of ADHD symptoms, but a reduction of depression symptoms was reported. Bloch et al. (2008) conducted an open label rTMS study with nine adolescents (16-18 age range) affected by severe treatment-resistant depression. Participants received 14 sessions of $10 \mathrm{~Hz}$ rTMS to the left DLPFC. Depression scores of the participants improved significantly. Two of them remained in clinical remission 1 year after therapy (as judged by regular clinical follow-up). Five subjects reported mild headaches. No other adverse effects were reported. More recently, Wall et al. (2011) conducted an open-label trial of adjunctive rTMS in eight adolescents with treatment-resistant depression. These subjects were maintained on a stable dose of a selective serotonin reuptake inhibitor (SSRI). All in all, thirty daily $10 \mathrm{~Hz}$ rTMS treatments were given over the left dorsolateral prefrontal cortex. The CDRS-Revised mean scores improved significantly from baseline over the course of 30 treatments and the 6-month follow-up. Pre- and post-treatment neurocognitive testing did not reveal any decline in functioning. These data are preliminary as no control group was included, but this study shows that intensive treatment parameters were well tolerated by adolescent patients. No studies for treatment of depression in children have been conducted with tDCS.

The available studies suggest some therapeutic effect of rTMS for the treatment of childhood depression. A recurring element associated with a successful rTMS therapy is the stimulation of left DLPFC at $10 \mathrm{~Hz}$. However, since these are open and/or case report studies, the results of these studies should be interpreted with caution.

\section{SCHIZOPHRENIA}

Childhood-onset schizophrenia is a rare and severe form of the disorder (Nicolson and Rapoport, 1999), that is neurobiologically and physiologically continuous with adult onset schizophrenia (David et al., 2011). Hallucinations are, probably, the most dramatic clinical symptom that causes significant problems for the life of patients with schizophrenia.

Physiological abnormalities have been reported in several neural regions including right medial temporal, lateral temporal, inferior frontal cortex, and in the cingulate cortex bilaterally, left DLPFC and left superior temporal gyrus (Vyas and Gogtay, 2012; Hayempour et al., 2013). However, neuroimaging studies have shown relatively less predictive value despite consistent reports of progressive structural brain abnormalities associated with schizophrenia. An increase of left temporoparietal cortex excitability is associated with positive symptoms, specifically auditory hallucinations (AHs) (Silbersweig et al., 1995; Shergill et al., 2000). On the other hand, activity enhancement of the left prefrontal region has been suggested to improve negative symptoms (Heimer et al., 1997), due to its effect on the release of dopamine (Strafella et al., 2001). Therefore, excitability-reducing stimulation is proposed to reduce activity of the left temporoparietal cortex, while excitatory non-invasive brain stimulation has been used to increase left prefrontal region activation for therapeutic application (see Freitas et al., 2009; Demirtas-Tatlidede et al., 2013 for a complete review).
Jardri et al. (2007) report a single case study involving a 11year-old boy with medication-resistant schizophrenia. An fMRI scan displayed increased auditory cortex activity with concurrent AHs. 10 sessions of $1 \mathrm{~Hz}$ rTMS were administered to the left temporoparietal cortex. Verbal AHs decreased by $50 \%$. The improvement obtained with rTMS was maintained by repeating the sessions every 5 weeks. No adverse effects of rTMS were reported. More recently, the same group (Jardri et al., 2012) established a case series of adolescents diagnosed with childhood-onset schizophrenia according to the Schedule for Affective Disorders and Schizophrenia for School-Age Children $(n=10,15.5$ years old). All participants had frequent and drug-resistant AHs. The patients received $1 \mathrm{~Hz}$ rTMS to the T3-P3 site over 5 days. The authors assessed scalp discomfort clinically and describe only minor discomfort. AHRS scores decreased significantly from baseline to the immediate post-treatment assessment, and from baseline to the 1-month assessment. Furthermore, the Global Assessment of Functioning scores improved significantly immediately and 1 month after the treatment, as compared to baseline values.

For tDCS, one study was recently published with regard to childhood-onset schizophrenia (Mattai et al., 2011). This study aimed to investigate the tolerability of tDCS in this patient group. Twelve participants ( 12 children, age range 10-17) were assigned to one of two groups: bilateral anodal DLPFC stimulation $(n=8)$ or bilateral cathodal superior temporal gyrus (STG) stimulation $(n=5)$. The stimulation protocol consisted of $20 \mathrm{~min}$ per day, per 2 weeks. No subjects reported significant discomfort at the electrode sites. However, four individuals had transient redness of the skin under the electrodes that resolved within about an hour after treatment. Although no significant clinical improvement has been reported, this study is the first to demonstrate that tDCS with the applied parameters is well tolerated in adolescents. Complains of fatigue reported by some patients could be related to unspecific effects, such as medication regimens that frequently include the atypical antipsychotic clozapine.

Taken together, knowledge about the effects of non-invasive brain stimulation in childhood onset schizophrenia is still limited. However, the rTMS case reports provided by Jardri et al. hint for some efficacy in reducing hallucinations. The only available tDCS study is in favor for good tolerability of this stimulation protocol. Systematic studies are needed to explore this field to a larger degree.

\section{DISCUSSION}

In this work we reviewed the available literature about the effects of non-invasive brain stimulation in pediatric populations (i.e., younger than 18 years) suffering from neuro-psychiatric diseases. We focused our analysis on studies which have examined the therapeutic efficacy of rTMS and tDCS for rehabilitating neurological functions and ameliorating psychiatric syndromes in children.

In general, the studies provide preliminary evidence in support for a therapeutic potential of non-invasive stimulation techniques in children and adolescents. However, several limitations should be taken into account.

Virtually no double-blinded sham controlled studies are available at present, which makes it difficult to make safe statements 


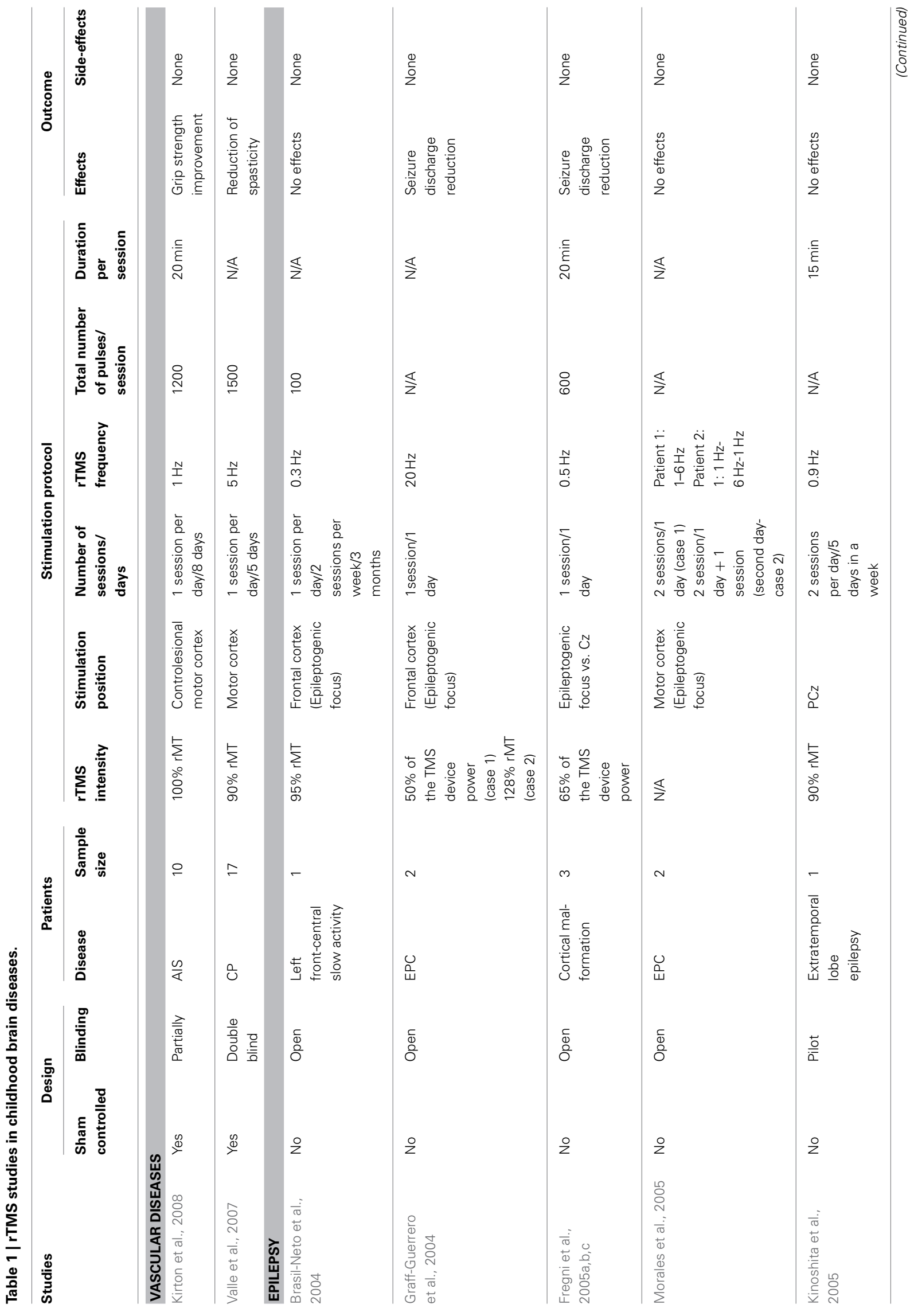




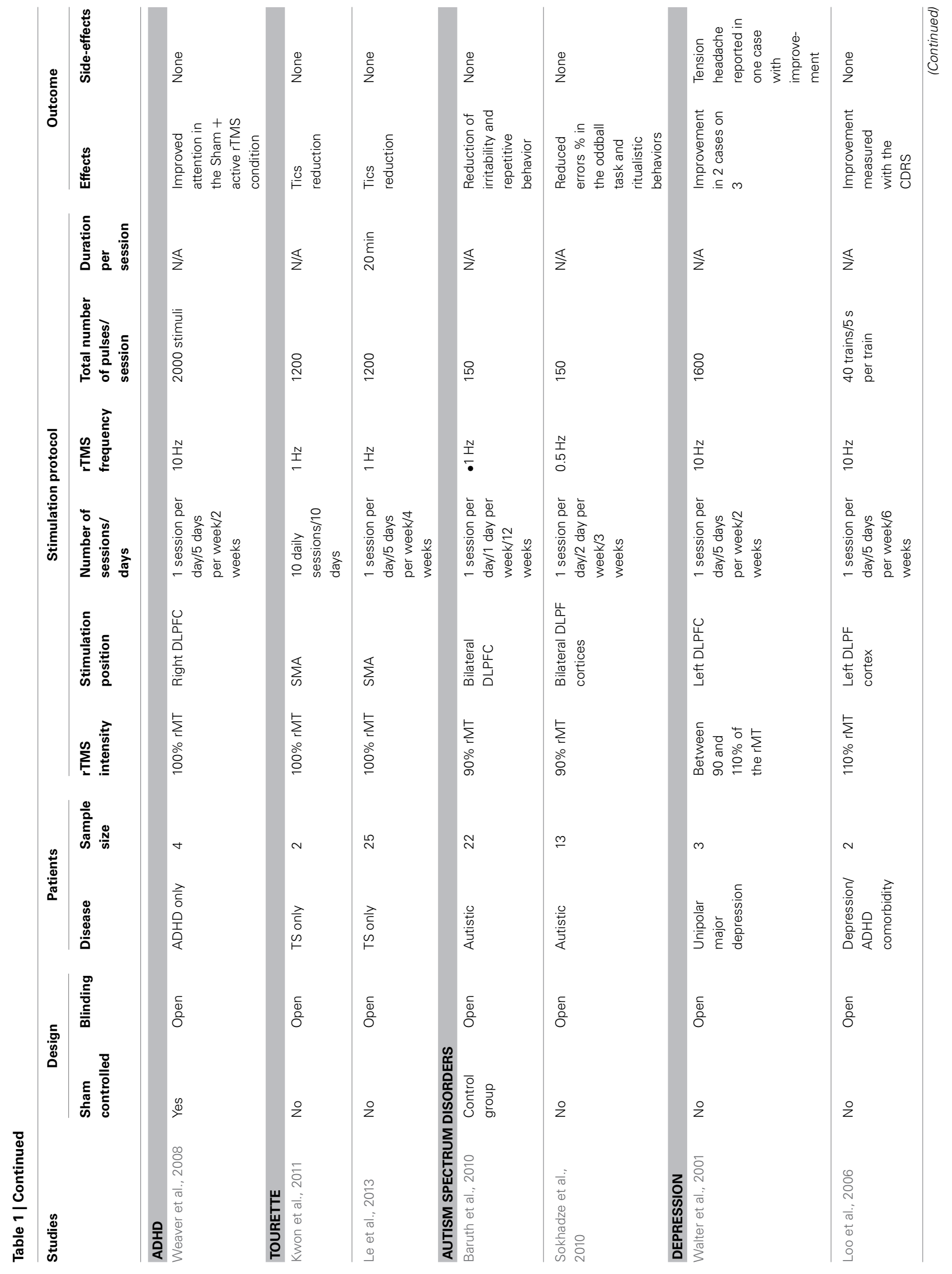




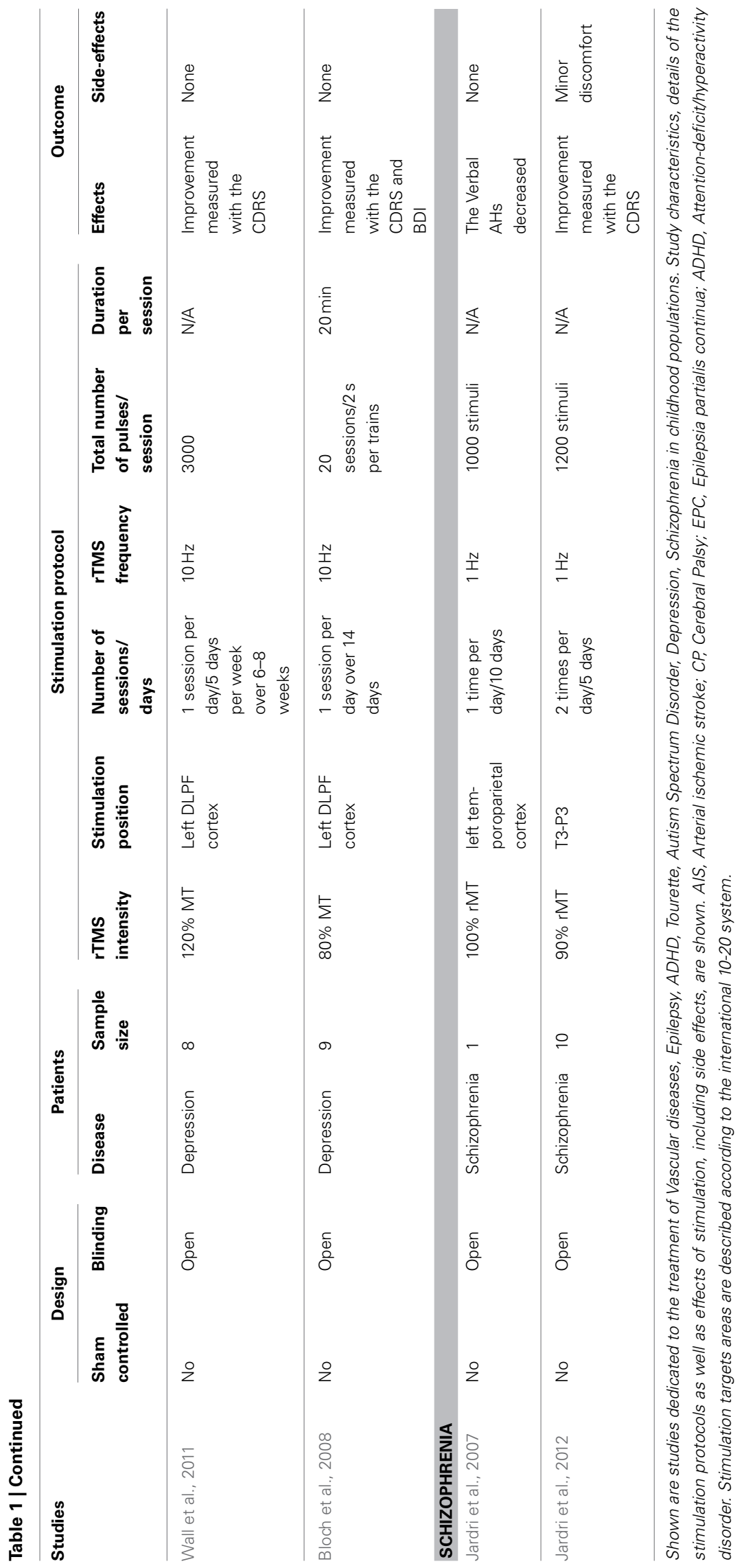




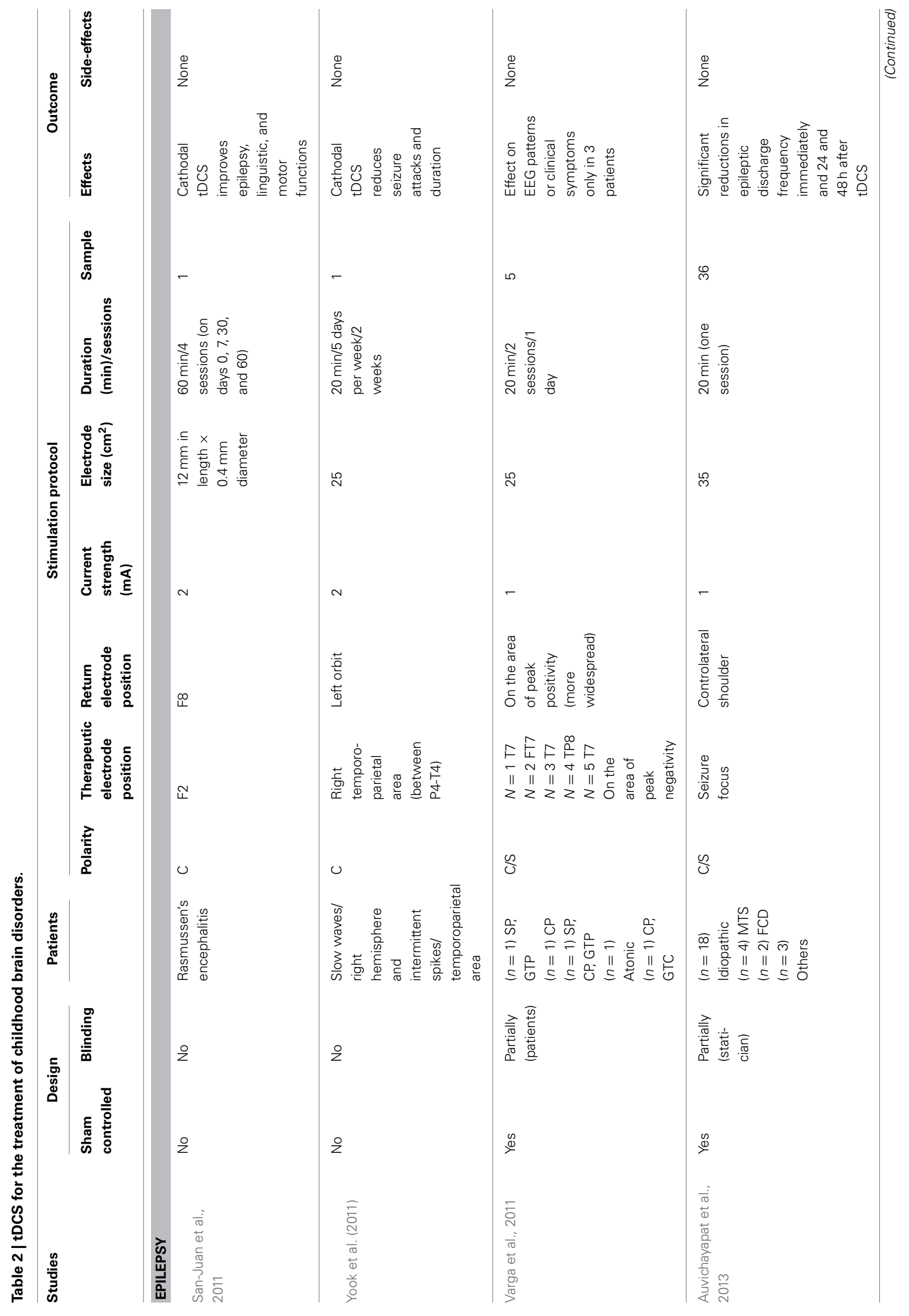




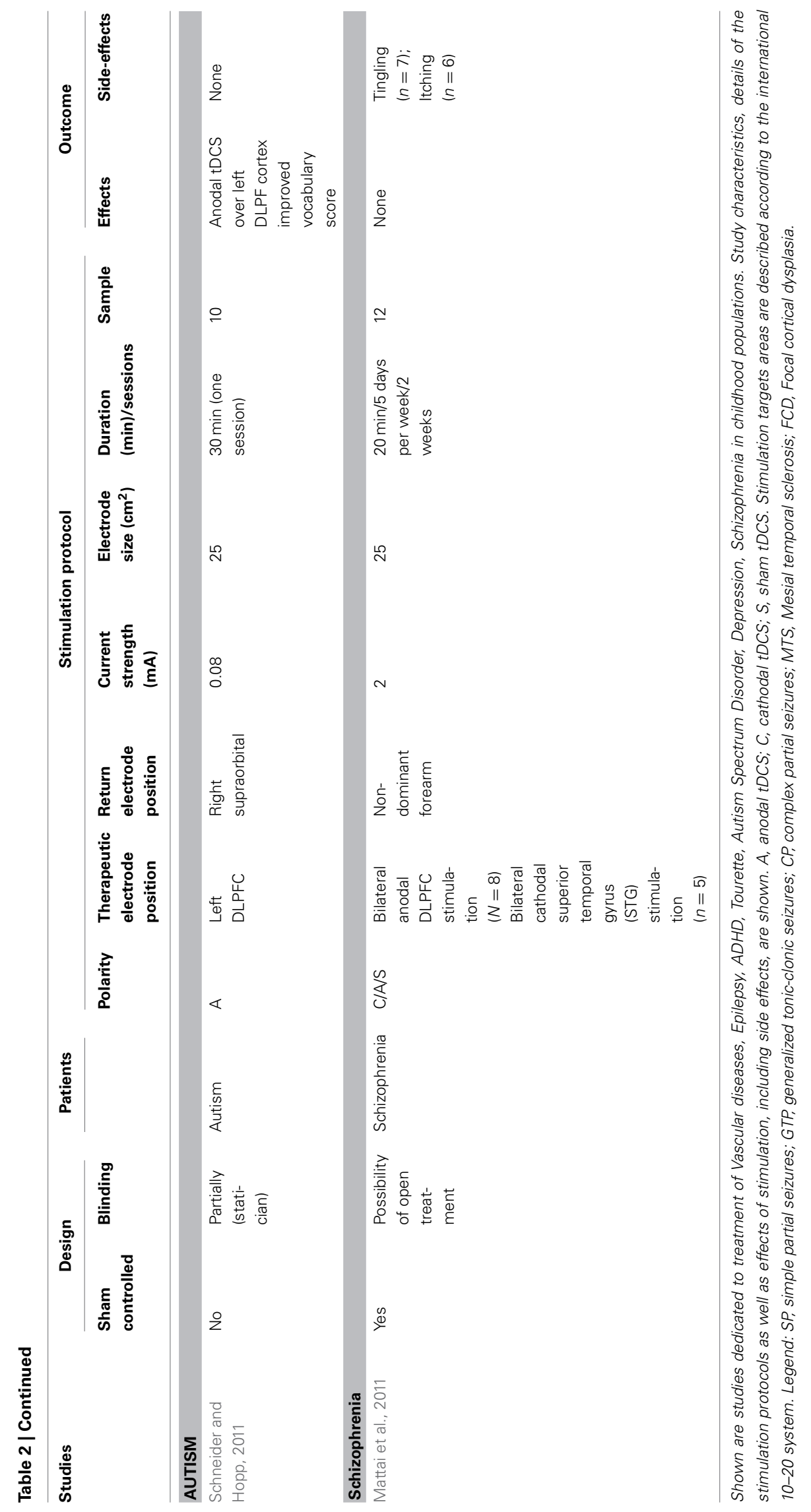


about efficacy. Since there seems to be a potential, these studies are urgently needed. Moreover, systematic studies to identify optimal stimulation protocols based on physiology are missing, including comparisons between different protocols, and a systematic evaluation of safety of non-invasive brain stimulation with regard to the developing brain. It is important to note that, although the examined literature does deliver no hint for major adverse effects in children, the evidence currently available is still rather scanty. Studies conducted in adult humans and animal models are probably not transferable one-to-one to children, since anatomy and physiology differs relevantly between these groups (Johnston, 2009). In this regard, it is important to consider that the developing brain is characterized by "sensitive" periods or rather periods during which the effects of the experience on the brain are unusually strong during a limited period in development (Knudsen, 2004). This suggests that, during development, the risk to induce maladaptive neural plasticity due to brain stimulation techniques might be relatively high (Vicario and Nitsche, 2013). Because of this, we suggest that neuroscientists willing to apply non-invasive brain stimulation in children should consider dose-finding studies, and a longitudinal monitoring of neural plasticity triggered by these methods (through neuroimaging and/or electrophysiological techniques), to control for functional and structural changes. In this connection, it will be important to improve knowledge about physiological effects of stimulation in children. This might provide important landmarks for assessing the therapeutic effectiveness of the adopted stimulation protocol as well as the presence of side effects. Moreover, discoveries provided by research in "sensitive" periods at the circuit level might be helpful for this issue as they could supply insights of paramount importance for predicting the trajectories of neural plasticity induced by non-invasive brain stimulation techniques and their related therapeutic results. Worthy of discussion is also the fact that the current state of investigations involving the application of brain stimulation methods in pediatric populations is unbalanced toward TMS (See Tables 1, 2 for detailed summaries).

No published studies are currently available with regard to the therapeutic efficacy of tDCS in childhood disorders such as stroke and/or brain injury, TS, ADHD, and Depression. Nevertheless, the interest in applying tDCS for treating pediatric populations is growing.

\section{FUTURE DIRECTION OF RESEARCH}

The application of non-invasive brain stimulation for the rehabilitation and/or treatment of children might have the potential advantage of promoting improvements superior to those achievable in adulthood, according to the fact that the developing brain is characterized by "sensitive periods" during which the effects of the experience on the brain are unusually strong for a limited period (Knudsen, 2004). Therefore, the improvement of symptoms via non-invasive stimulation treatment in pediatric age might be more stable, and consistent. However, as discussed above, this same "sensitivity" might also be risky in terms of induction of maladaptive neural plasticity.

In order to minimize this risk, future lines of research should address the following, still limited, field of investigations:
- Exploration, also by using animal models, of the physiological effects of non-invasive brain stimulation in children;

- Systematic exploration of childhood clinical populations to clarify the pathophysiology of the respective diseases, which could be objective to non-invasive brain stimulation. This is important for defining optimal protocols.

- Systematic conduction of "dose-finding," sham-controlled, double-blinded studies, which will provide important information not available from the open studies.

These aspects will help to clarify, at several levels of complexity, the potential therapeutic efficacy of non-invasive brain stimulation in children, delivering a realistic basis for clinical application of such stimulation protocols on a large scale.

\section{REFERENCES}

American Academy of Pediatrics. (2000). Clinical practice guidelines: diagnosis and evaluation of the child with attention-deficit/hyperactivity disorder. Pediatrics 105, 1158-1170. doi: 10.1542/peds.105.5.1158

Auvichayapat, N., Rotenberg, A., Gersner, R., Ngodklang, S., Tiamkao, S., Tassaneeyakul, W., et al. (2013). Transcranial direct current stimulation for treatment of refractory childhood focal epilepsy. Brain Stimul. 6, 696-700. doi: 10.1016/j.brs.2013.01.009

Baruth, J. M., Casanova, M. F., El-Baz, A., Horrell, T., Mathai, G., Sears, L., et al. (2010). Low-frequency repetitive transcranial magnetic stimulation (rTMS) modulates evoked-gamma frequency oscillations in autism spectrum disorder (ASD). J. Neurother. 14, 179-194. doi: 10.1080/10874208.2010.501500

Bloch, Y., Grisaru, N., Harel, E. V., Beitler, G., Faivel, N., Ratzoni, G., et al. (2008). Repetitive transcranial magnetic stimulation in the treatment of depression in adolescents: an open-label study. J. ECT 24, 156-159. doi: 10.1097/YCT.0b013e318156aa49

Brasil-Neto, J. P., de Araújo, D. P., Teixeira, W. A., Araújo, V. P., and Boechat-Barros, R. (2004). Experimental therapy of epilepsy with transcranial magnetic stimulation: lack of additional benefit with prolonged treatment. Arq. Neuropsiquiatr. 62, 21-25. doi: 10.1590/S0004-282X2004000100004

Brazzo, D., Pera, M. C., Fasce, M., Papalia, G., Balottin, U., and Veggiotti, P. (2012). Epileptic encephalopathies with status epilepticus during sleep: new techniques for understanding pathophysiology and therapeutic options. Epilepsy Res. Treat. 2012, 642-725. doi: 10.1155/2012/642725

Bush, G. (2011). Cingulate, frontal, and parietal cortical dysfunction in attention-deficit/hyperactivity disorder. Biol. Psychiatry 69, 1160-1167. doi: 10.1016/j.biopsych.2011.01.022

Cosgrove, V. E., Roybal, D., and Chang, K. D. (2013). Bipolar depression in pediatric populations: epidemiology and management. Paediatr. Drugs 15, 83-91. doi: 10.1007/s40272-013-0022-8

Croarkin, P. E., Wall, C. A., and Lee, J. (2011). Applications of transcranial magnetic stimulation (TMS) in child and adolescent psychiatry. Int. Rev. Psychiatry 23, 445-453. doi: 10.3109/09540261.2011.623688

David, C. N., Greenstein, D., Clasen, L., Gochman, P., Miller, R., Tossell, J. W., et al. (2011). Childhood onset schizophrenia: high rate of visual hallucinations. J. Am. Acad. Child Adolesc. Psychiatry 50, 681.e3-686.e3. doi: 10.1016/j.jaac. 2011.03.020

Demirtas-Tatlidede, A., Vahabzadeh-Hagh, A. M., and Pascual-Leone, A. (2013). Can noninvasive brain stimulation enhance cognition in neuropsychiatric disorders? Neuropharmacology 64, 566-578. doi: 10.1016/j.neuropharm.2012. 06.020

Dudek, F. E., and Sutula, T. P. (2007). Epileptogenesis in the dentate gyrus: a critical perspective. Prog. Brain Res. 163, 755-773. doi: 10.1016/S0079-6123(07) 63041-6

Fitzgerald, P. B., Oxley, T. J., Laird, A. R., Kulkarni, J., Egan, G. F., and Daskalakis, Z. J. (2006). An analysis of functional neuroimaging studies of dorsolateral prefrontal cortical activity in depression. Psychiatry Res. 148, 33-45. doi: 10.1016/j.pscychresns.2006.04.006

Flöel, A. (2013). tDCS-enhanced motor and cognitive function in neurological diseases. Neuroimage doi: 10.1016/j.neuroimage.2013.05.098. [Epub ahead of print]. 
Flöel, A., Rösser, N., Michka, O., Knecht, S., and Breitenstein, C. (2008). Non invasive brain stimulation improves language learning. J. Cogn. Neurosci. 8, 1415-1422. doi: 10.1162/jocn.2008.20098

Fombonne, E. (2009). Epidemiology of pervasive developmental disorders. Pediatr. Res. 65, 591-598. doi: 10.1203/PDR.0b013e31819e7203

Freitas, C., Farzan, F., and Pascual-Leone, A. (2013). Assessing brain plasticity across the lifespan with transcranial magnetic stimulation: why, how, and what is the ultimate goal? Front. Neurosci. 7:42. doi: 10.3389/fnins.2013.00042

Freitas, C., Fregni, F., and Pascual-Leone, A. (2009). Meta-analysis of the effects of repetitive transcranial magnetic stimulation (rTMS) on negative and positive symptoms in schizophrenia. Schizophr. Res. 108, 11-24. doi: 10.1016/j.schres.2008.11.027

Fregni, F., Boggio, P. S., Nitsche, M., Bermpohl, F., Antal, A., Feredoes, E., et al. (2005a). Anodal transcranial direct current stimulation of prefrontal cortex enhances working memory. Exp. Brain Res. 166, 23-30. doi: 10.1007/s00221005-2334-6

Fregni, F., Boggio, P. S., Mansur, C. G., Wagner, T., Ferreira, M. J., Lima, M. C., et al. (2005b). Transcranial direct current stimulation of the unaffected hemisphere in stroke patients. Neuroreport 16, 1551-1555. doi: 10.1097/01.wnr. $000177010.44602 .5 \mathrm{c}$

Fregni, F., Thome-Souza, S., Bermpohl, F., Marcolin, M. A., Herzog, A., PascualLeone, A., et al. (2005c). Antiepileptic effects of repetitive transcranial magnetic stimulation in patients with cortical malformations: an EEG and clinical study. Stereotact. Funct. Neurosurg. 83, 57-62. doi: 10.1159/000086674

García-Larrea, L., Lukaszewicz, A. C., and Mauguière, F. (1992). Revisiting the oddball paradigm. Non-target vs neutral stimuli and the evaluation of ERP attentional effects. Neuropsychologia 30, 723-741. doi: 10.1016/0028-3932(92) 90042-K

Gaudeau-Bosma, C., Moulier, V., Allard, A. C., Sidhoumi, D., Bouaziz, N., Braha, S., et al. (2013). Effect of two weeks of rTMS on brain activity in healthy subjects during an n-back task: a randomized double blind study. Brain Stimul. 6, 569-575. doi: 10.1016/j.brs.2012.10.009

George, M. S., Sallee, F. R., Nahas, Z., Oliver, N. C., and Wassermann, E. M. (2001). Transcranial magnetic stimulation (TMS) as a research tool in Tourette syndrome and related disorders. Adv. Neurol. 85, 225-235.

Graff-Guerrero, A., Gonzáles-Olvera, J., Ruiz-García, M., Avila-Ordoñez, U., Vaugier, V., and García-Reyna, J. C. (2004). rTMS reduces focal brain hyperperfusion in two patients with EPC. Acta Neurol. Scand. 109, 290-296. doi: 10.1046/j.1600-0404.2003.00222.x

Grefkes, C., and Fink, G. R. (2011). Reorganization of cerebral networks after stroke: new insights from neuroimaging with connectivity approaches. Brain 134, 1264-1276. doi: 10.1093/brain/awr033

Hamilton, R. H., Wiener, M., Drebing, D. E., and Coslett, H. B. (2013). Gone in a flash: manipulation of audiovisual temporal integration using transcranial magnetic stimulation. Front. Psychol. 4:571. doi: 10.3389/fpsyg.2013.00571

Hayempour, B. J., Newberg, A., and Alavi, A. (2013). Neuromolecular imaging instrumentation demonstrating dysfunctional brain function in schizophrenic patients. J. Alzheimers Dis. Parkinsonism 3, pii: 114. doi: 10.4172/2161-0460. 1000114

Heimer, L., Harlan, R. E., Alheid, G. F., Garcia, M. M., and de Olmos, J. (1997). Substantia innominata: a notion which impedes clinical-anatomical correlations in neuropsychiatric disorders. Neuroscience 76, 957-1006. doi: 10.1016/S0306-4522(96)00405-8

Jardri, R., Bubrovszky, M., Demeulemeester, M., Poulet, E., Januel, D., Cohen, D., et al. (2012). Repetitive transcranial magnetic stimulation to treat early-onset auditory hallucinations. J. Am. Acad. Child Adolesc. Psychiatry 51, 947-949. doi: 10.1016/j.jaac.2012.06.010

Jardri, R., Lucas, B., Delevoye-Turrell, Y., Delmaire, C., Delion, P., Thomas, P., et al. (2007). An 11-year-old boy with drug-resistant schizophrenia treated with temporo-parietal rTMS. Mol. Psychiatry 12, 320. doi: 10.1038/sj.mp.4001968

Johnston, M. V. (2009). Plasticity in the developing brain: implications for rehabilitation. Dev. Disabil. Res. Rev. 15, 94-101. doi: 10.1002/ddrr.64

Kammer, T., and Spitzer, M. (2012). Brain stimulation in psychiatry: methods and magnets, patients and parameters. Curr. Opin. Psychiatry 25, 535-541. doi: 10.1097/YCO.0b013e328358df8c

Kinoshita, M., Ikeda, A., Begum, T., Yamamoto, J., Hitomi, T., and Shibasaki, H. (2005). Low-frequency repetitive transcranial magnetic stimulation for seizure suppression in patients with extratemporal lobe epilepsy-a pilot study. Seizure 14, 387-392. doi: 10.1016/j.seizure.2005.05.002
Kirton, A., Chen, R., Friefeld, S., Gunraj, C., Pontigon, A. M., and Deveber, G. (2008). Contralesional repetitive transcranial magnetic stimulation for chronic hemiparesis in subcortical paediatric stroke: a randomised trial. Lancet Neurol. 7, 507-513. doi: 10.1016/S1474-4422(08)70096-6

Knudsen, E. I. (2004). Sensitive periods in the development of the brain and behavior. J. Cogn. Neurosci. 16, 1412-1425. doi: 10.1162/0898929042304796

Krause, B., Márquez-Ruiz, J., and Cohen Kadosh, R. (2013). The effect of transcranial direct current stimulation: a role for cortical excitation/inhibition balance? Front. Hum. Neurosci. 7:602. doi: 10.3389/fnhum.2013.00602

Kuo, M. F., and Nitsche, M. A. (2012). Effects of transcranial electrical stimulation on cognition. Clin. EEG Neurosci. 43, 192-199. doi: 10.1177/1550059412444975

Kuo, M. F., Paulus, W., and Nitsche, M. A. (2013). Therapeutic effects of noninvasive brain stimulation with direct currents (tDCS) in neuropsychiatric diseases. Neuroimage doi: 10.1016/j.neuroimage.2013.05.117. [Epub ahead of print].

Kwon, H. J., Lim, W. S., Lim, M. H., Lee, S. J., Hyun, J. K., Chae, J. H., et al. (2011). 1-Hz low frequency repetitive transcranial magnetic stimulation in children with Tourette's syndrome. Neurosci. Lett. 492, 1-4. doi: 10.1016/j.neulet.2011.01.007

Lakatos, P., Karmos, G., Mehta, A. D., Ulbert, I., and Schroeder, C. E. (2008). Entrainment of neuronal oscillations as a mechanism of attentional selection. Science 320, 110-113. doi: 10.1126/science.1154735

Le, K., Liu, L., Sun, M., and Xiao, N. (2013). Transcranial magnetic stimulation at 1 Hertz improves clinical symptoms in children with Tourette syndrome for at least 6 months. J. Clin. Neurosci. 20, 257-262. doi: 10.1016/j.jocn.2012. 01.049

Lee, J., Ku, J., Han, K., Park, J., Lee, H., Kim, K. R., et al. (2013). rTMS over bilateral inferior parietal cortex induces decrement of spatial sustained attention. Front. Hum. Neurosci. 7:26. doi: 10.3389/fnhum.2013.00026

Loo, C., McFarquhar, T., and Walter, G. (2006). Transcranial magnetic stimulation in adolescent depression. Australas. Psychiatry 14, 81-85. doi: 10.1111/j.14401665.2006.02251.x

Mattai, A., Miller, R., Weisinger, B., Greenstein, D., Bakalar, J., Tossell, J., et al. (2011). Tolerability of transcranial direct current stimulation in childhoodonset schizophrenia. Brain Stimul. 4, 275-280. doi: 10.1016/j.brs.2011.01.001

Mayberg, H. S. (2007). Defining the neural circuitry of depression: toward a new nosology with therapeutic implications. Biol. Psychiatry 61, 729-730. doi: 10.1016/j.biopsych.2007.01.013

McFadden, K. L., Hepburn, S., Winterrowd, E., Schmidt, G. L., and Rojas, D. C. (2012). Abnormalities in gamma-band responses to language stimuli in firstdegree relatives of children with autism spectrum disorder: an MEG study. BMC Psychiatry 12:213. doi: 10.1186/1471-244X-12-213

Morales, O. G., Henry, M. E., Nobler, M. S., Wassermann, E. M., and Lisanby, S. H. (2005). Electroconvulsive therapy and repetitive transcranial magnetic stimulation in children and adolescents: a review and report of two cases of epilepsia partialis continua. Child Adolesc. Psychiatr. Clin. N. Am. 14, 193-210. doi: 10.1016/j.chc.2004.07.010

Naeser, M. A., Martin, P. I., Nicholas, M., Baker, E. H., Seekins, H., HelmEstabrooks, N., et al. (2005). Improved naming after TMS treatments in a chronic, global aphasia patient-case report. Neurocase 11, 182-193. doi: 10.1080/13554790590944663

Nicolson, R., and Rapoport, J. L. (1999). Childhood-onset schizophrenia: rare but worth studying. Biol. Psychiatry 46, 1418-1428. doi: 10.1016/S00063223(99)00231-0

Nitsche, M. A., Cohen, L. G., Wassermann, E. M., Priori, A., Lang, N., Antal, A., et al. (2008). Transcranial direct current stimulation: state of the art 2008. Brain Stimul. 1, 206-223. doi: 10.1016/j.brs.2008.06.004

Nitsche, M. A., and Paulus, W. (2009). Noninvasive brain stimulation protocols in the treatment of epilepsy: current state and perspectives. Neurotherapeutics 6, 244-250. doi: 10.1016/j.nurt.2009.01.003

Nitsche, M. A., and Paulus, W. (2011). Transcranial direct current stimulationupdate 2011. Restor. Neurol. Neurosci. 29, 463-492. doi: 10.3233/RNN-20110618

Nitsche, M. A., Schauenburg, A., Lang, N., Liebetanz, D., Exner, C., Paulus, W., et al. (2003). Facilitation of implicit motor learning by weak transcranial direct current stimulation of the primary motor cortex in the human. J. Cogn. Neurosci. 15, 619-626. doi: 10.1162/089892903321662994

O’Reardon, J. P., Solvason, H. B., Janicak, P. G., Sampson, S., Isenberg, K. E., Nahas, Z., et al. (2007). Efficacy and safety of transcranial magnetic stimulation in the 
acute treatment of major depression: a multisite randomized controlled trial. Biol. Psychiatry 62, 1208-1216. doi: 10.1016/j.biopsych.2007.01.018

Pal, P. K., Hanajima, R., Gunraj, C. A., Li, J. Y., Wagle-Shukla, A., Morgante, F., et al. (2005). Effect of low-frequency repetitive transcranial magnetic stimulation on interhemispheric inhibition. J. Neurophysiol. 94, 1668-1675. doi: 10.1152/jn.01306.2004

Pascual-Leone, A., Amedi, A., Fregni, F., and Merabet, L. B. (2005). The plastic human brain cortex. Annu. Rev. Neurosci. 28, 377-401. doi: 10.1146/annurev. neuro.27.070203.144216

Pascual-Leone, A., Rubio, B., Pallardó, F., and Catalá, M. D. (1996). Rapidrate transcranial magnetic stimulation of left dorsolateral prefrontal cortex in drug-resistant depression. Lancet 348, 233-237. doi: 10.1016/S01406736(96)01219-6

Pascual-Leone, A., Valls-Solé, J., Brasil-Neto, J. P., Cammarota, A., Grafman, J., and Hallett, M. (1994). Akinesia in Parkinson's disease. II. Effects of subthreshold repetitive transcranial motor cortex stimulation. Neurology 44, 892-898. doi: 10.1212/WNL.44.5.892

Picard, N., and Strick, P. L. (2001). Imaging the premotor areas. Curr. Opin. Neurobiol. 11, 663-672. doi: 10.1016/S0959-4388(01)00266-5

Quartarone, A., Bagnato, S., Rizzo, V., Morgante, F., Santangelo, A., Battaglia, F., et al. (2005). Distinct changes in cortical and spinal excitability following highfrequency repetitive TMS to the human motor cortex. Exp. Brain Res. 161, 114-124. doi: 10.1007/s00221-004-2052-5

Quintana, H. (2005). Transcranial magnetic stimulation in persons younger than the age of 18. J. ECT. 21, 88-95. doi: 10.1097/01.yct.0000162556.02720.58

San-Juan, D., Calcáneo, J. D., González-Aragón, M. F., Bermúdez Maldonado, L., Avellán, A. M., Argumosa, E. V., et al. (2011). Transcranial direct current stimulation in adolescent and adult Rasmussen's encephalitis. Epilepsy Behav. 20, 126-131. doi: 10.1016/j.yebeh.2010.10.031

Schneider, H. D., and Hopp, J. P. (2011). The use of the Bilingual Aphasia Test for assessment and transcranial direct current stimulation to modulate language acquisition in minimally verbal children with autism. Clin. Linguist. Phon. 25, 640-654. doi: 10.3109/02699206.2011.570852

Shergill, S. S., Brammer, M. J., Williams, S. C., Murray, R. M., and McGuire, P. K. (2000). Mapping auditory hallucinations in schizophrenia using functional magnetic resonance imaging. Arch. Gen. Psychiatry 57, 1033-1038. doi: 10.1001/archpsyc.57.11.1033

Silbersweig, D. A., Stern, E., Frith, C., Cahill, C., Holmes, A., Grootoonk, S., et al. (1995). A functional neuroanatomy of hallucinations in schizophrenia. Nature 378, 176-179. doi: 10.1038/378176a0

Sokhadze, E., Baruth, J., Tasman, A., El-Baz, A., Mansoor, M., Ramaswamy, R., et al. (2010). Low-frequency repetitive transcranial magnetic stimulation (rTMS) affects event-related potential measures of novelty processing in autism. Appl. Psychophysiol. Biofeedback 35, 147-161. doi: 10.1007/s10484-0099121-2

Stafstrom, C. E. (2006). Neonatal seizures: is a novel, mechanism-based treatment finally on the horizon? Epilepsy Curr. 6, 130-132. doi: 10.1111/j.15357511.2006.00121.x

Stagg, C. J., and Nitsche, M. A. (2011). Physiological basis of transcranial direct current stimulation. Neuroscientist 17, 37-53. doi: 10.1177/1073858410386614

Strafella, A. P., Paus, T., Barrett, J., and Dagher, A. (2001). Repetitive transcranial magnetic stimulation of the human prefrontal cortex induces dopamine release in the caudate nucleus. J. Neurosci. 21, RC157.

Swain, J. E., Scahill, L., Lombroso, P. J., King, R. A., and Leckman, J. F. (2007) Tourette syndrome and tic disorders: a decade of progress. J. Am. Acad. Child Adolesc. Psychiatry 46, 947-968. doi: 10.1097/chi.0b013e318068fbcc

Takeuchi, N., Chuma, T., Matsuo, Y., Watanabe, I., and Ikoma, K. (2005). Repetitive transcranial magnetic stimulation of contralesional primary motor cortex improves hand function after stroke. Stroke 36, 2681-2686. doi: 10.1161/01.STR.0000189658.51972.34

Tallon-Baudry, C., Bertrand, O., Peronnet, F., and Pernier, J. (1998). Induced gamma-band activity during the delay of a visual short-term memory task in humans. J. Neurosci. 18, 4244-4254.
Tanoue, R. T., Jones, K. T., Peterson, D. J., and Berryhill, M. E. (2013). Differential frontal involvement in shifts of internal and perceptual attention. Brain Stimul. 6, 675-682. doi: 10.1016/j.brs.2012.11.003

Tavabi, K., Embick, D., and Roberts, T. P. (2011). Spectral-temporal analysis of cortical oscillations during lexical processing. Neuroreport 22, 474-478. doi: 10.1097/WNR.0b013e3283476b84

Valle, A. C., Dionisio, K., Pitskel, N. B., Pascual-Leone, A., Orsati, F., Ferreira, M. J., et al. (2007). Low and high frequency repetitive transcranial magnetic stimulation for the treatment of spasticity. Dev. Med. Child Neurol. 49, 534-538. doi: 10.1111/j.1469-8749.2007.00534.x

Varga, E. T., Terney, D., Atkins, M. D., Nikanorova, M., Jeppesen, D. S., and Uldall, P. (2011). Transcranial direct current stimulation in refractory continuous spikes and waves during slow sleep: a controlled study. Epilepsy Res. 97, 142-145. doi: 10.1016/j.eplepsyres.2011.07.016

Vicario, C. M., Candidi, M., and Aglioti, S. M. (2013a). Cortico-spinal embodiment of newly acquired, action-related semantic associations. Brain Stimul. doi: 10.1016/j.brs.2013.05.010. [Epub ahead of print].

Vicario, C. M., Martino, D., and Koch, G. (2013b). Temporal accuracy and variability in the left and right posterior parietal cortex. Neuroscience 245, 121-128. doi: 10.1016/j.neuroscience.2013.04.041

Vicario, C. M., Martino, D., Spata, F., Defazio, G., Giacchè, R., Martino, V., et al. (2010). Time processing in children with Tourette's syndrome. Brain Cogn. 73, 28-34. doi: 10.1016/j.bandc.2010.01.008

Vicario, C. M., and Nitsche, M. A. (2013). Transcranial direct current stimulation: a remediation tool for the treatment of childhood congenital dyslexia? Front Hum. Neurosci. 7:139. doi: 10.3389/fnhum.2013.00139

Vicario, C. M., and Rumiati, R. I. (2012). tDCS of the primary motor cortex improves the detection of semantic dissonance. Neurosci. Lett. 518, 133-137. doi: 10.1016/j.neulet.2012.04.070

Vyas, N. S., and Gogtay, N. (2012). Treatment of early onset schizophrenia: recent trends, challenges and future considerations. Front. Psychiatry 3:29. doi: 10.3389/fpsyt.2012.00029

Wall, C. A., Croarkin, P. E., Sim, L. A., Husain, M. M., Janicak, P. G., Kozel, F. A., et al. (2011). Adjunctive use of repetitive transcranial magnetic stimulation in depressed adolescents: a prospective, open pilot study. J. Clin. Psychiatry 72, 1263-1269. doi: 10.4088/JCP.11m07003

Walter, G., Tormos, J. M., Israel, J. A., and Pascual-Leone, A. (2001). Transcranial magnetic stimulation in young persons: a review of known cases. J. Child Adolesc. Psychopharmacol. 11, 69-75. doi: 10.1089/104454601750143483

Weaver, L., Mace, W., Akhtar, U., Moss, E., Rostain, A., and O’Reardon, J. (2008). Safety and efficacy of rTMS in treatment of ADHD in adolescents and young persons. J. ECT 24, 105-106.

Yook, S. W., Park, S. H., Seo, J. H., Kim, S. J., and Ko, M. H. (2011). Suppression of seizure by cathodal transcranial direct current stimulation in an epileptic patient - a case report -. Ann. Rehabil. Med. 35, 579-582. doi: 10.5535/arm.2011.35.4.579

Conflict of Interest Statement: The authors declare that the research was conducted in the absence of any commercial or financial relationships that could be construed as a potential conflict of interest.

Received: 19 September 2013; accepted: 06 November 2013; published online: 25 November 2013.

Citation: Vicario CM and Nitsche MA (2013) Non-invasive brain stimulation for the treatment of brain diseases in childhood and adolescence: state of the art, current limits and future challenges. Front. Syst. Neurosci. 7:94. doi: 10.3389/fnsys.2013.00094 This article was submitted to the journal Frontiers in Systems Neuroscience. Copyright (C) 2013 Vicario and Nitsche. This is an open-access article distributed under the terms of the Creative Commons Attribution License (CC BY). The use, distribution or reproduction in other forums is permitted, provided the original author(s) or licensor are credited and that the original publication in this journal is cited, in accordance with accepted academic practice. No use, distribution or reproduction is permitted which does not comply with these terms. 